\title{
Conditions of equivalence of effects for the solid body from incompressible material
}

\author{
Elephan Agakhanov ${ }^{1, *}$, Murad Agakhanov ${ }^{2}$, Lyudmila Sultanova ${ }^{1}$, and Zabiya Hizriyeva ${ }^{1}$ \\ ${ }^{1}$ Dagestan State Technical University, Imam Shamil Ave. 70, 367015 Makhachkala, Russia \\ ${ }^{2}$ Moscow State University of Civil Engineering, Yaroslavskoe shosse, 26, Moscow, 129337, Russia
}

\begin{abstract}
Polemicizing with the existing opinion that modern numerical methods allow to solve practically any problem of mechanics, it should be noted that analytical and experimental methods still are relevant, and a complex of methods leads to development of mechanics of a deformable solid body. At present one of the most important directions of development of mechanics of a deformable solid body is creation of the approaches that allow to combine organically great computing opportunities of modern supercomputers with experimental methods of the material and design research. In engineering practice at production of designs and products incompressible materials are widely used. Assessment of their durability requires detailed studying of deflected mode caused by action of various loadings and forces. For a solid body from incompressible material, using the resolving equations set of mechanics of a deformable solid body, at action of the compelled deformations of a general view, volume and superficial forces conditions of equivalence are established. It is shown that the known solutions are special cases of the established equivalence conditions. The efficiency of the analytical solution of a three-dimensional task on the rotating disk from incompressible material is shown by the method of equivalence of effects.
\end{abstract}

\section{Introduction}

At production of various designs and products or their elements in engineering practice incompressible materials are widely used. For assessment of their durability it is necessary to study in detail the deflected mode caused by action of various loadings and forces $[1,2]$.

At the solution of tasks in mechanics of a deformable solid body, especially when using experimental methods, conditions of equivalence of influences are effectively applied [3, 4]. Therefore establishment of conditions of equivalence of effects for incompressible material has fundamental value in the theory of calculation of designs and products or their elements from incompressible material.

For the first time in the method of equivalence of effects offered by S.P. Tymoshenko it has been shown that at the solution of problems of thermoelasticity the compelled deformations caused by the temperature field formally can be replaced with the volume

\footnotetext{
* Corresponding author: muradak@mail.ru
} 
forces included into the balance equations in movements and the superficial loadings included into boundary conditions in tension. At the same time movements and tangent tension in the initial and replacing tasks coincide, and normal tension differs at some known value. Further theoretical development of this method was undertaken by various authors, for example, N.F. Lebedev [5] for incompressible material.

As theoretical solutions of tasks at various effects have comparable difficulty, the greatest application the method of equivalence of influences has found in pilot studies, mainly, in a polarizing and optical method. Polemicizing with the existing opinion that modern numerical methods allow to solve practically any problem of mechanics, it should be noted that analytical and experimental methods still are relevant, especially a complex of methods that leads to development of mechanics of a deformable solid body $[6,7,8]$. At present one of the most important directions of development of mechanics of a deformable solid body is creation of the approaches allowing to combine organically enormous computing opportunities of modern supercomputers with experimental methods of a research of materials and designs $[9,10,11]$.

\section{State of the problem}

Let us consider the body of an arbitrary form of incompressible material occupying the spatial area $V$ limited by the closed surface $\Gamma=\Gamma_{u}+\Gamma_{\sigma}$. Let the intense deformed state in a body be caused by action of the compelled deformations $\bar{\varepsilon}_{i j}(t)$, superficial forces $P_{i}(t)$ on the site of border $\Gamma_{\sigma}$, volume forces $\Gamma_{\sigma}$ and the shift $f_{i}(t)$ of points of a part of the border $\Gamma_{u}$.

\section{Method of research}

In the case under consideration the resolving system of the equations includes $[12,13]$ : balance equations:

$$
\begin{aligned}
& \nabla^{2} U_{i}(t)+ \frac{1}{3} \frac{\partial}{\partial i}\left[e(t)+2 \bar{e}(t)+\frac{\theta(t)}{G(t)}\right]+\int_{\tau_{1}}^{t}\left\{\nabla^{2} U_{i}(\tau)+\frac{1}{3} \frac{\partial}{\partial i}[e(\tau)+2 \bar{e}(\tau)]\right\} \times \\
& \times \frac{\partial}{\partial \tau} \Delta_{1}(t, \tau) d \tau-2\left\{\sum_{j} \frac{\partial \bar{\varepsilon}_{i j}(t)}{\partial j}+\int_{\tau_{1}}^{t}\left[\sum_{j} \frac{\partial \bar{\varepsilon}_{i j}(\tau)}{\partial j}\right] \frac{\partial}{\partial \tau} \Delta_{1}(t, \tau) d \tau\right\}+(1) \\
&+\frac{F_{i}(t)}{G(t)}=0 \text { in } V,
\end{aligned}
$$

boundary conditions:

$$
\sum_{j}\left\{2 G ( t ) \left\{\varepsilon_{i j}(t)-\bar{\varepsilon}_{i j}(t)-\frac{e(t)-\bar{e}(t)}{3} \delta_{i j}+\int_{\tau_{1}}^{t} \frac{\partial}{\partial \tau} \Delta_{1}(t, \tau)\left[\varepsilon_{i j}(\tau)-\bar{\varepsilon}_{i j}(\tau)-\right.\right.\right.
$$




$$
\begin{gathered}
\left.\left.\left.-\frac{e(\tau)-\bar{e}(\tau)}{3} \delta_{i j}\right] d \tau\right\}+\frac{\delta_{i j}}{3} \theta(t)\right\} n_{j}=P_{i}(t) \text { on } \Gamma_{\sigma}, \\
U_{i}(t)=f_{i}(t) \text { on } \Gamma_{u},
\end{gathered}
$$

incompressibility condition:

$$
e(t)=\bar{e}(t) .
$$

From (1) - (4) we have the following condition of identical equality of movements $U_{i}^{(P)}(t) \equiv U_{i}^{(F, \bar{\varepsilon})}(t)$ :

$$
\begin{gathered}
\sum_{j}\left\{\frac{\partial \bar{\varepsilon}_{i j}(t)}{\partial i}-\left\{\sum_{j} \frac{\partial \bar{\varepsilon}_{i j}(t)}{\partial j}+\int_{\tau_{1}}^{t}\left[\sum_{j} \frac{\partial \bar{\varepsilon}_{i j}(\tau)}{\partial j}\right] \frac{\partial}{\partial \tau} \Delta_{1}(t, \tau) d \tau-\frac{3}{2 E(t)} F_{i}(t)\right\} \times\right. \\
\left.\times \delta_{i j}+\int_{\tau_{1}}^{t} \frac{\partial \bar{\varepsilon}_{i j}(\tau)}{\partial i} \frac{\partial}{\partial \tau} \Delta_{1}(t, \tau) d \tau\right\} n_{j}=\frac{3}{2 E(t)} \frac{\partial P_{i}(t)}{\partial i} .
\end{gathered}
$$

The dependence connecting tension with deformations is of the form [1]:

$$
\begin{gathered}
\sigma_{i j}(t)=2 G(t)\left\{\varepsilon_{i j}(t)-\bar{\varepsilon}_{i j}(t)-\frac{e(t)-\bar{e}(t)}{3} \delta_{i j}+\int_{\tau_{1}}^{t} \frac{\partial}{\partial \tau} \Delta_{1}(t, \tau)\left[\varepsilon_{i j}(\tau)-\bar{\varepsilon}_{i j}(\tau)-\right.\right. \\
\left.\left.-\frac{e(\tau)-\bar{e}(\tau)}{3} \delta_{i j}\right] d \tau\right\}+\frac{\delta_{i j}}{3} \theta(t) .
\end{gathered}
$$

From (6) for incompressible material on condition of identical equality of movements $U_{i}^{(P)}(t) \equiv U_{i}^{(F, \bar{\varepsilon})}(t)$ we have:

$$
\begin{aligned}
\sigma_{i j}^{(P)}(t)= & \sigma_{i j}^{(F, \bar{\varepsilon})}(t)+\frac{2 E(t)}{3}\left\{\bar{\varepsilon}_{i j}(t)-\frac{\delta_{i j}}{n_{i}}\left\{\sum _ { j } \left[\bar{\varepsilon}_{i j}(t)+\int_{\tau_{1}}^{t} \frac{\partial}{\partial \tau} \Delta_{1}(t, \tau) \times\right.\right.\right. \\
& \left.\left.\left.\times \bar{\varepsilon}_{i j}(\tau) d \tau\right] n_{j}-\frac{3 P_{i}(t)}{2 E(t)}\right\}+\int_{\tau_{1}}^{t} \frac{\partial}{\partial \tau} \Delta_{1}(t, \tau) \bar{\varepsilon}_{i j}(\tau) d \tau\right\} .
\end{aligned}
$$

For bigger clarity of the ratio (5) and (7) we will write down once in more detail:

$$
\begin{aligned}
& \left\{\frac{\partial \bar{\varepsilon}_{x y}(t)}{\partial y}+\frac{\partial \bar{\varepsilon}_{x z}(t)}{\partial z}+\int_{\tau_{1}}^{t}\left[\frac{\partial \bar{\varepsilon}_{x y}(\tau)}{\partial y}+\frac{\partial \bar{\varepsilon}_{x z}(\tau)}{\partial z}\right] \frac{\partial}{\partial \tau} \Delta_{1}(t, \tau) d \tau\right\} n_{x}- \\
& -\left[\frac{\partial \bar{\varepsilon}_{x y}(t)}{\partial x}+\int_{\tau_{1}}^{t} \frac{\partial \bar{\varepsilon}_{x y}(\tau)}{\partial x} \frac{\partial}{\partial \tau} \Delta_{1}(t, \tau) d \tau\right] n_{y}-\left[\frac{\partial \bar{\varepsilon}_{x z}(t)}{\partial x}+\int_{\tau_{1}}^{t} \frac{\partial \bar{\varepsilon}_{x z}(\tau)}{\partial x} \times\right.
\end{aligned}
$$




$$
\begin{gathered}
\left.\times \frac{\partial}{\partial \tau} \Delta_{1}(t, \tau) d \tau\right] n_{z}=\frac{3}{2 E(t)}\left[F_{x}(t) n_{x}-\frac{\partial P_{x}(t)}{\partial x}\right], \\
\sigma_{x x}^{(P)}(t)=\sigma_{x x}^{(F)}(t)+\sigma_{x x}^{(\bar{\varepsilon})}(t)+\frac{2 E(t)}{3}\left\{\bar{\varepsilon}_{x x}(t)-\frac{1}{n_{x}}\left[\bar{\varepsilon}_{x x}(t) n_{x}+\right.\right. \\
+\bar{\varepsilon}_{x y}(t) n_{y}+\bar{\varepsilon}_{x z}(t) n_{z}+\int_{\tau_{1}}^{t} \bar{\varepsilon}_{x x}(\tau) \frac{\partial}{\partial \tau} \Delta_{1}(t, \tau) d \tau n_{x}+ \\
+\int_{\tau_{1}}^{t} \bar{\varepsilon}_{x y}(\tau) \frac{\partial}{\partial \tau} \Delta_{1}(t, \tau) d \tau n_{y}+\int_{\tau_{1}}^{t} \bar{\varepsilon}_{x z}(\tau) \frac{\partial}{\partial \tau} \Delta_{1}(t, \tau) d \tau n_{z}- \\
\left.\left.-\frac{3}{2 E(t)} P_{x}(t)\right]+\int_{\tau_{1}}^{t} \bar{\varepsilon}_{x x}(\tau) \frac{\partial}{\partial \tau} \Delta_{1}(t, \tau) d \tau\right\}, \\
\sigma_{x y}^{(P)}(t)=\sigma_{x y}^{(F)}(t)+\sigma_{x y}^{(\bar{\varepsilon})}(t)+\frac{2 E(t)}{3}\left[\bar{\varepsilon}_{x y}(t)+\int_{\tau_{1}}^{t} \bar{\varepsilon}_{x y}(\tau) \frac{\partial}{\partial \tau} \Delta_{1}(t, \tau) d \tau\right]
\end{gathered}
$$

Let the tensor of the compelled deformations be spherical, i.e.

$$
\bar{\varepsilon}_{x x}(t)=\bar{\varepsilon}_{y y}(t)=\bar{\varepsilon}_{z z}(t)=\xi(t), \quad \bar{\varepsilon}_{x y}(t)=\bar{\varepsilon}_{x z}(t)=\bar{\varepsilon}_{y z}(t)=0 .
$$

Then

$$
\xi(t)=0 .
$$

Taking into account (11) expressions (8) - (10) take a form:

$$
\begin{gathered}
F_{x}(t) n_{x}=\frac{\partial P_{x}(t)}{\partial x} \text { or } \frac{\partial P(t)}{\partial x}=F_{x}(t) . \\
\sigma_{x x}^{(P)}(t)=\sigma_{x x}^{(F)}(t)+\frac{P_{x}(t)}{n_{x}}=\sigma_{x x}^{(F)}(t)+P(t), \\
\sigma_{x y}^{(P)}(t)=\sigma_{x y}^{(F)}(t) .
\end{gathered}
$$

In case of an elastic body the expressions (8) - (10) take a form:

$$
\begin{gathered}
{\left[\frac{\partial \bar{\varepsilon}_{x y}(t)}{\partial y}+\frac{\partial \bar{\varepsilon}_{x z}(t)}{\partial z}\right] n_{x}-\frac{\partial \bar{\varepsilon}_{x y}(t)}{\partial x} n_{y}-\frac{\partial \bar{\varepsilon}_{x z}(t)}{\partial x} n_{z}=} \\
=\frac{3}{2 E(t)}\left[F_{x}(t) n_{x}-\frac{\partial P_{x}(t)}{\partial x}\right]
\end{gathered}
$$




$$
\begin{gathered}
\sigma_{x x}^{(P)}(t)=\sigma_{x x}^{(F)}(t)+\sigma_{x x}^{(\bar{\varepsilon})}(t)+\frac{2 E(t)}{3}\left\{\bar{\varepsilon}_{x x}(t)-\frac{1}{n_{x}}\left[\bar{\varepsilon}_{x x}(t) n_{x}+\bar{\varepsilon}_{x y}(t) n_{y}+\right.\right. \\
\left.\left.+\bar{\varepsilon}_{x z}(t) n_{z}-\frac{3}{2 E(t)} P_{x}(t)\right]\right\}, \\
\sigma_{x y}^{(P)}(t)=\sigma_{x y}^{(F)}(t)+\sigma_{x y}^{(\bar{\varepsilon})}(t)+\frac{2 E(t)}{3} \bar{\varepsilon}_{x y}(t) .
\end{gathered}
$$

In case of a spherical tensor of the compelled deformations the expressions (15)-(17) coincide with the expressions (12)-(14) received for an inelastic body.

\section{Analysis of results}

As an example we will consider the analytical solution of a three-dimensional task on the rotating disk from incompressible material by the method of equivalence of effects. The round disk of constant thickness from incompressible material is under the influence of volume centrifugal forces

$$
F_{x}=\rho \omega^{2} x, \quad F_{y}=\rho \omega^{2} y, \quad F_{z}=0,
$$

where: $\rho$ - mass of volume unit,

$\omega$ - angular speed of a disk.

At the solution of a three-dimensional task on the rotating S.P. Tymoshenko's disk, at first we find the private solution satisfying the equations of balance and conditions of compatibility and then impose the solution in the form of a polynom of the fifth degree and select constants in it in order to meet boundary conditions of the problem.

Tensions $\sigma_{z}^{(F)}$ and $\tau_{r z}^{(F)}$ in a disk from volume centrifugal forces is equal to zero (flat tension), and tensions $\sigma_{r}^{(F)}$ and $\sigma_{\theta}^{(F)}$ change on thickness of a disk and are functions of $r$ and $z$.

Then, according to the equivalence of effects established in work in case of incompressibility of material of a disk, we have

$$
\begin{aligned}
& \sigma_{z}^{(F)}=\sigma_{z}^{(P)}-\sigma_{z}^{(\xi)}-P, \\
& \tau_{r z}^{(F)}=\tau_{r z}^{(P)}-\tau_{r z}^{(\xi)}, \\
& \sigma_{r}^{(F)}=\sigma_{r}^{(P)}-P, \\
& \sigma_{\theta}^{(F)}=\sigma_{\theta}^{(P)}-P .
\end{aligned}
$$

where the replacing superficial forces $P$ and the compelled deformations $\xi$ have an appearance

$$
P=\frac{\rho \omega^{2} r^{2}}{2},
$$




$$
\xi=\frac{\rho \omega^{2} r^{2}}{4 E} .
$$

For definition of tension of the disk which is under the influence of the replacing superficial forces it is possible to use function of tension in the form of a polynom of the fifth degree [5]

$$
\varphi=a_{5}\left(8 z^{5}-40 r^{2} z^{3}+15 r^{4} z\right)+b_{5}\left(2 z^{5}-r^{2} z^{3}-3 r^{4} z\right)
$$

Expressions for components of tension taking into account (4) have an appearance

$$
\begin{gathered}
\sigma_{r}^{(p)}=-a_{5}\left(180 r^{2}-240 z^{2}\right)+b_{5}\left(9 r^{2}+60 z^{2}\right), \\
\sigma_{z}^{(p)}=-a_{5}\left(-240 r^{2}+480 z^{2}\right)+b_{5}\left(42 z^{2}-75 r^{2}\right), \\
\sigma_{\theta}^{(p)}=a_{5}\left(-60 r^{2}+240 z^{2}\right)+b_{5}\left(60 z^{2}-15 r^{2}\right), \\
\tau_{r z}^{(p)}=480 a_{5} r z-42 b_{5} r z .
\end{gathered}
$$

Defining constants $a_{5}$ и $b_{5}$ in such a way that at $z= \pm c$ or $r=a$ $\tau_{r z}^{(p)}=0$ and at $z= \pm c \quad \sigma_{z}^{(p)}=P$, we get

$$
\begin{aligned}
& \sigma_{r}^{(p)}=\rho \omega^{2}\left[\frac{1}{16} r^{2}-\frac{3}{4} z^{2}\right], \\
& \sigma_{\theta}^{(p)}=\rho \omega^{2}\left[\frac{3}{16} r^{2}-\frac{3}{4} z^{2}\right] .
\end{aligned}
$$

In order to satisfy a boundary condition on a disk contour for radial tension $\sigma_{r}^{(p)}$, i.e. to achieve performance of a condition

$$
\left(\int_{-c}^{c} \sigma_{r}^{(p)} d z\right)_{r=a}=\rho \omega^{2} a^{2} c
$$

let us impose on tension (24) uniform radial stretching of the value

$$
\rho \omega^{2}\left[\frac{7}{16} a^{2}+\frac{3}{4} \frac{c^{2}}{3}\right] \text {. }
$$

Then full tension from the replacing superficial forces will be defined by formulas

$$
\sigma_{r}^{(p)}=\rho \omega^{2}\left[\frac{1}{16} r^{2}+\frac{1}{4}\left(c^{2}-3 z^{2}\right)+\frac{7}{16} a^{2}\right],
$$




$$
\sigma_{\theta}^{(p)}=\rho \omega^{2}\left[\frac{3}{16} r^{2}+\frac{1}{4}\left(c^{2}-3 z^{2}\right)+\frac{7}{16} a^{2}\right] .
$$

Substituting (20) and (25) in (19) we receive the solution of an initial task

$$
\begin{aligned}
\sigma_{r}^{(F)} & =\rho \omega^{2}\left[\frac{7}{16}\left(a^{2}-r^{2}\right)+\frac{1}{4}\left(c^{2}-3 z^{2}\right)\right], \\
\sigma_{\theta}^{(F)} & =\rho \omega^{2}\left[\frac{7}{16} a^{2}-\frac{5}{16} r^{2}+\frac{1}{4}\left(c^{2}-3 z^{2}\right)\right] .
\end{aligned}
$$

If in expressions for tension received by S.P. Tymoshenko we accept $v=0,5$, then they coincide with (26) that demonstrates reliability of the received solution. As the solution (26) is received with the use only of function of tension, in case of incompressibility of material of a disk it is effective to use equivalence of effects.

\section{Conclusions}

For a solid body from incompressible material, using the resolving system of the equations of mechanics of a deformable solid body, at action of the compelled deformations of a general view, volume and superficial forces conditions of equivalence both for tension, and for movements are established. It is shown that the known solutions are special cases of the established equivalence conditions. The efficiency of the analytical solution of a threedimensional task on the rotating disk from incompressible material is shown by the method of equivalence of effects.

\section{References}

1. S.V. Litvinov, E.S. Klimenko, I.I. Kulinich, B.M. Yazyev, The Bulletin of the Moscow State University of Civil Engineering, 2, 153-157 (2011)

2. S.V. Litvinov, B.M. Yazyev, A.N. Beskopylny, I.V. Ananyev, Electronic Scientific Magazine "Engineering Bulletin of the Don", 1 (2012)

3. G.E. Agakhanov, The Bulletin of the Dagestan State Technical University, 1, 8-16 (2015)

4. E.K. Agakhanov, M.K. Agakhanov, News of Higher Education Institutions. North Caucasus Region. Technical Science, 1, 25-26 (2005)

5. N.F. Lebedev, Application-oriented Mechanics, 2, 63-68 (1977)

6. G.E. Agakhanov, The Online Magazine "NAUKOVEDENIYE", 7 (2015)

7. V.I. Andreev, A.S. Avershyev, International Journal for Computational Civil and Structural Engi-neering, 9.3, 14-20 (2013)

8. V.I. Andreev, A.S. Avershyev, Applied Mechanics and Materials Vols, 580-583, 812815 (2014)

9. A.I. Akayev, M.M. Payzulayev, The Scientific Review, 5, 112-115 (2015)

10. A.I. Akayev, M.M. Payzulayev, The Scientific Review, 9, 87-90 (2015)

11. M. G. Magomedov, M.M. Payzulayev, The Scientific Review, 1, 77-81 (2015)

12. G.E. Agakhanov, The Scientific Review, 12, 733-736 (2014) 
13. E.K. Agakhanov, The Bulletin of the Dagestan State Technical University, 2, 39-45 (2013) 